\title{
Is an implant removal after dorsal plating of distal radius fracture always needed?
}

\author{
Gajdos R, Bozik M, Stranak P \\ FD Roosevelt Teaching Hospital Banska Bystrica, Department of Trauma Surgery, Banska Bystrica, Slovakia. \\ rgajdos@nspbb.sk
}

\begin{abstract}
PURPOSE: The purpose of this study was to evaluate the need for an implant removal and the frequency of hardware related complications in a group of distal radius fractures treated with dorsal double plating.

METHODS: This retrospective study analyzed data from 37 patients in whom distal radius fracture surgery was performed through single dorsal approach with two low profile titanium plates during a period of 5 years. Objective and subjective parameters were evaluated, focusing on detection of potentially harmful complications and the need of hardware removal during the follow up at least 12 months after the surgery.

RESULTS: 37 patients with distal radius fracture treated by dorsal double plating were included in this study. 19 patients had their implant removed and 18 patients had their implant retained. There was no statistical difference between these groups in respect to age, fracture type, incidence of associated wrist injury, hand dominance, gender and Mayo wrist score. Only subjective parameters including the average value of the Quick Disabilities of Arm, Hand, and Shoulder Questionnaire and the Visual Analogue Scale were higher in the group of patient requiring implant removal with a statistically significant difference.

CONCLUSION: Our results suggested that only symptomatic hardware should be removed. A decreased range of motion and extensor tendon problems are the most frequent complication, that necessitate plate removal. The main indicator of implant related soft tissue problems was the dorsal wrist pain syndrome (Tab. 1, Fig. 3, Ref. 36). Text in PDF www.elis.sk.
\end{abstract}

KEY WORDS: distal radius fracture, dorsal plates, implant removal.

\section{Introduction}

Distal radius fractures are one of the most frequent fractures in the upper extremity (1). With increasing requirements for the best possible function after these fractures, not only in young patients, but also in the group of elderly, treatment of these fractures has undergone considerable changes over the past decades (2). While patients with low functional demands still may be treated by nonoperative means, high demand patients, regardless their age, may require surgical fixation (3). According to a general consensus (4, 5) articular step off more than $2 \mathrm{~mm}$, radial shortening of $>2-4$ $\mathrm{mm}$, radial inclination $>5-15^{\circ}$, sagittal tilt on lateral view $>15^{\circ}$ dorsal and $>20^{\circ}$ volar, are indications for a surgical intervention. The goals of surgical treatment are anatomical reduction, stable fixation and early rehabilitation (6). Anatomical reduction includes reduction and reconstruction of the articular surface and restoration of axes and the length $(7,8,9,10,11,12,13,14)$.

F.D. Roosevelt Teaching Hospital Banska Bystrica, Department of Trauma Surgery, Banska Bystrica, Slovakia

Address for correspondence: R. Gajdos, MD, FD Roosevelt Teaching Hospital, Banska Bystrica, Department of Trauma Surgery, Namestie L. Svobodu 1, SK-974 01 Banska Bystrica, Slovakia. Phone: +421.48.4412157, Fax: +421.48.441 2162

Acknowledgment: The authors thank Mária Konderová for statistical analysis and Zuzana Polishook and Veronika Gajdos for correcting the English grammar.
Although meta-analysis has failed to show superiority of specific operative treatment method $(15)$, Jupiter $(3,16)$ and other researchers $(5,17,18)$ acknowledged that open reduction and internal fixation with plates has obvious advantages over the other methods of distal radius fracture fixation. It allows a direct restoration of anatomy, stable internal fixation, shorter immobilization period, and thus an earlier return of wrist function.

Due to a high complication rate after dorsal plating, there is a tendency to use volar approach and volar plates for fixation of distal radius fractures during the last years (3). Despite this trend, there are still some indications for dorsal open reduction and plate fixation. These indications include intraarticular fractures with impacted articular fragments, displaced dorso-ulnar fragment, intraarticular fractures with an associated proximal carpal row bony or ligamentous injury (6).

Despite the development of new low profile implants, tendon irritation or rupture remains one of the most frequent complication after dorsal plating (19). Tendon rupture has been reported as early as 8 weeks and as late as 7 months after the surgery (20). As a result of these complications, the dorsal implants often have to be removed (21). Indications for implant removal are not well established. Some authors suggest early implant removal to avoid possible complications $(22,23)$, others remove only symptomatic hardware $(24,25)$.

Hardware removal has significant economic implications including the direct costs of the procedure as well as indirect costs 
of possible work time lost for postoperative recovery. Implant removal may be challenging and lead to complications as well (26).

This paper analyzes the frequency and reasons for implant removal in distal radius fractures treated through single dorsal approach. It also analyzes parameters which may help to identify patients that may profit from metal removal and those where hardware retaining is appropriate.

\section{Materials and methods}

In this retrospective study we analyzed data from 236 patients with distal radius fracture treated operatively from January 2007 to December 2011 by a single surgeon in F. D. Roosevelt Teaching Hospital in Banská Bystrica, Slovakia. All surgeries and follow up examinations were performed by a senior surgeon (R.G.). This study was approved by the institutional ethical committee.

Patients with distal radius fracture treated by single dorsal approach and dorsal plating were identified and enrolled to this study. Underlying pathology, for which dorsal approach was indicated, was recorded. Among these indications were distal radius fracture with impacted articular fragment, displaced dorso-ulnar fragment and associated proximal carpal row bony or ligamentous injury. Patients with an associated nerve or vascular injury, compartment syndrome, combined dorsal and volar approach, follow up shorter than 12 months and younger than 18 were excluded. All fractures were classified according to AO classification. In all patients age, gender, hand dominance, type of injury, injury to operation interval and associated bony or ligamentous injuries were recorded.

\section{Initial surgery}

All patients had their surgery within 3 weeks of injury. All fractures in study group were fixed with two LCP $2.4 \mathrm{~mm}$ dorsal distal radius titanium plates (Synthes, Switzerland) by technique similar to dorsal perpendicular double plate fixation technique described by Rikli et al in 2005 (6). Associated carpal injuries were repaired according to the type of injury. Ligament repair was protected by carpal bones Kirschner wire transfixation. At the end of the procedure, the first extensor compartment on radial side was left open. On dorso-ulnar side, tendons were left in a subcutaneous position with no direct contact to the dorso-ulnar plate. This plate was covered by radially based „U“-shaped retinacular flap. After the surgery, wrist was immobilized in a removable splint for a maximum of 6 weeks. After this period, Kirschner wires were removed in patients with carpal bone transfixation. The rehabilitation under the supervision of a physiotherapist targeted on wrist joint function followed.

\section{Follow-up}

All patients were followed up on regular basis at outpatient clinic for at least one year. During the follow-up visit, objective and subjective parameters were evaluated and recorded. Objective parameters included wrist's range of motion and radiographic evaluation, where loss of correction, development of posttraumatic wrist joint arthritis, signs of hardware loosening or prominence were assessed and recorded. Subjective parameters included patient re- ported score on the Quick Disabilities of Arm, Hand, and Shoulder questionnaire (Quick DASH) and a Visual Analogue Scale (VAS), as well as physician's rating system Mayo Wrist score (MWS). Complications including tenosynovitis or tendon rupture, as well as range of motion limitations caused by plate prominence or arthrofibrosis were recorded. In case of a suspicion of tendon irritation, the diagnosis was confirmed by an ultrasound examination.

During the follow-up period, an implant removal was indicated in case of: pronounced subjective inconvenience, clinical and radiological signs of hardware related complications.

\section{Hardware removal surgery}

All hardware removal procedures were performed under general anesthesia or peripheral nerve blockade. Tourniquet on upper arm was used and dorsal straight incision in original scar was made. Dissection of subcutaneous tissue, identification and retraction of extensor pollicis longus tendon and the superficial branch of the radial nerve was done. After identification of hardware location, locking head screws were removed first, cortical screw last. Plate was released from the underlying tissue and removed. The surgery was finished by the closure of wound in layers.

\section{Statistical analysis}

Hardware removal divided the study group into 2 subgroups. In the first subgroup were patients with retained hardware, in the second subgroup were patients with hardware removed. Based on medical records, these subgroups were comparable in relation to: age, gender, hand dominance, type of fracture, associated carpal injuries, VAS, Quick DASH and MWS. Based on this comparison, the predictors of hardware removal were proposed.

\section{Statistical methods}

Comparisons between the groups were done using an independent sample t-test for numerical variables (age, Quick DASH, VAS, MWS) and chi-square for nominal (gender, occupation, hand dominance, fracture type) ones. The significance level was set at $\mathrm{p}<0.05$ and power as $90 \%$ for all analyses.

\section{Results}

We identified 37 patients with distal radius fracture treated through single dorsal approach with a double plating technique during the period of 2007-2011, which met all inclusion criteria. This group of patients represented $15.7 \%$ of all distal radius fractures treated surgically during this period by a single surgeon.

Indications for single dorsal approach were: associated proximal carpal row ligamentous injury in 11 cases, associated proximal carpal row fracture in 6 cases, fracture pattern with impacted articular fragments or displaced dorso-ulnar fragment in 22 cases. According the AO classification, 29 fractures were C3 type, 5 fractures were $\mathrm{C} 2$ type and 3 fractures were B3 type. There were 26 males and 11 females, the mean age was 43.9 years (range 18-77). The mean injury to operation interval was 10.5 days (range 1-21). In four cases, bone graft with osteoconductive material was used to support articular surface in highly comminuted 


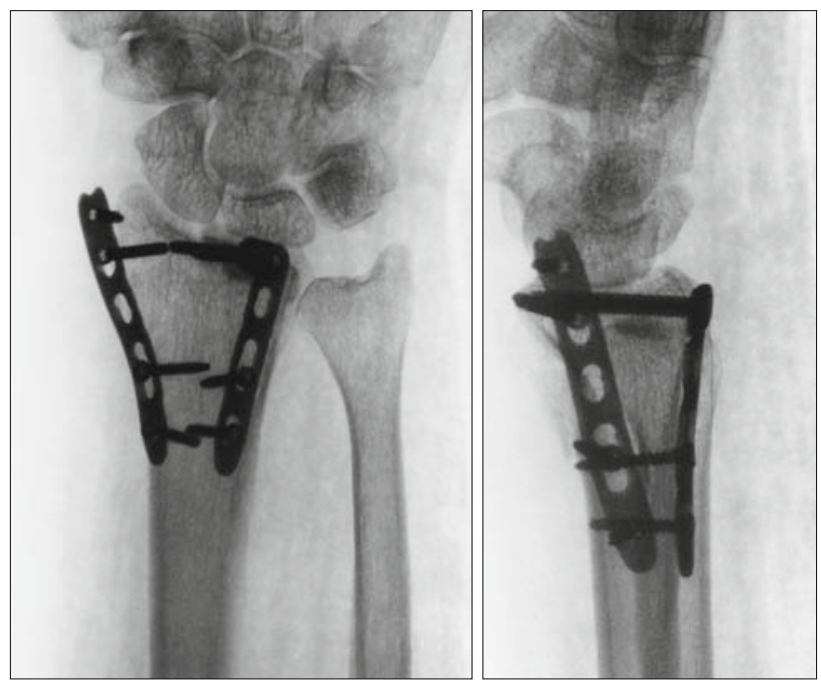

Fig. 1. Postoperative $X$-ray of the distal radius fracture treated with two dorsal plates through single dorsal approach. The prominence of radial lateral plate is evident.

fractures. All fractures finally healed with no signs of superficial or deep infection. The average follow up period was 17.1 months (range 12-41).

A high number of complications related directly to the dorsal plating was noticed. Two patients complained of superficial branch of the radial nerve dysesthesia. In one patient, the problem resolved completely after hardware removal and nerve release, the second one required chronic medication because of residual neuropathic pain. In one patient, the indication for metal removal was plate prominence resulting in radial wrist pain (Fig. 1). Further soft tissue complications including extensor tendon irritation or limited wrist range of motion were seen in 10 patients. All of them were treated with the implant removal and in case of range of motion restriction also by the manipulation under a general or peripheral block anesthesia.

In two cases, the implant removal was done during subsequent surgeries that were not directly related to the dorsal plating. In one patient it was done during the repair of missed TFCC

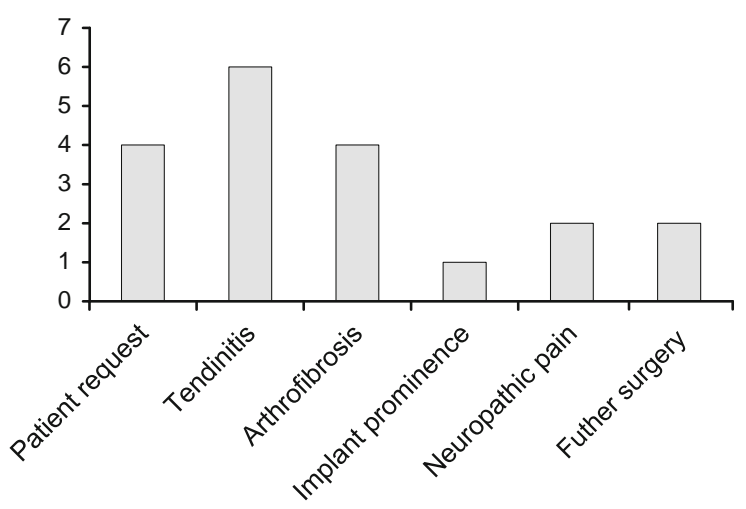

Fig. 2. Reasons for the implant removal with the amount of patients.

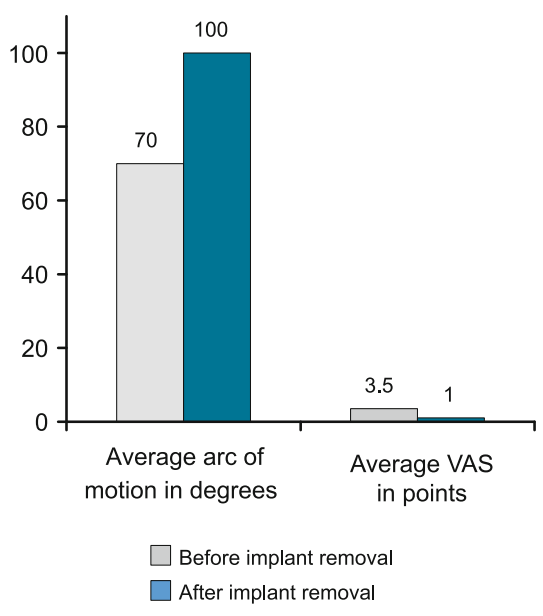

Fig. 3. Changes in range of motion and pain level after implant removal.

lesion and in another patient during the median nerve release. In this patient, median nerve neuropathy occurred 3 months after the surgery. Three patients required the implant removal on their own request and one patient was recommended the surgery due to her young age.

At the time of hardware removal, all plates were completely covered by the retinacular flap. Hypertrophy of the synovial tendon sheaths was present, but no tendon thinning or fraying was observed.

Finally, 19 patients $(51.3 \%)$ had their implant removed. The complete list of reasons is summarized in Figure 2. The average osteosynthesis to plate removal interval was 29 weeks (range 12-80). No complications related to the subsequent surgeries were observed. Patients who underwent implant removal because of dorsal wrist pain syndrome had their pain level decreased. In patients with wrist movement restrictions, the range of motion increased in all but one patient after the hardware removal and manipulation (Fig. 3). Patients who wanted implant removal on their own request or because of their age, overall 4 patients, were excluded from the statistical analysis, because there was no medical reason for the second surgery.

There was no significant difference between the subgroups with or without implant removal in respect to age, fracture type, incidence of associated wrist injuries, hand dominance and gender. Also the median Mayo wrist score was identical for both subgroups as 89 (range 75-100).

The average Quick DASH value was 19 (range 0-36) for the subgroup without hardware removal and 22 (range 9-36) for the subgroup with hardware removed. The difference of this variable between subgroups was statistically significant $(\mathrm{p}=0.018)$. Pain levels measured by Visual analogue scale were higher in the subgroup of patient requiring implant removal with a statistically significant difference $(p=0.0)$ (Tab. 1). No patient from the subgroup with a retained hardware enrolled in this study needed plate removal till the end of December 2013. 
Tab. 1. Statistical analysis.

\begin{tabular}{|c|c|c|c|c|}
\hline Characteristic & Retained plates group & Plates removal group & Test $\mathrm{p}$ value & Significance \\
\hline \multicolumn{5}{|l|}{ Gender } \\
\hline Males & 18 & 8 & Pearson Chi-Square & No significant difference \\
\hline Females & 4 & 7 & 0.063 & \\
\hline \multicolumn{5}{|l|}{ Age } \\
\hline Mean & 44.72 & 45.12 & t-test & No significant difference \\
\hline Range & $24-63$ & $31-77$ & 0.92 & \\
\hline \multicolumn{5}{|c|}{ Affected hand dominance } \\
\hline Dominant & 14 & 5 & Pearson Chi-Square & No significant difference \\
\hline Nondominant & 8 & 10 & 0.06 & \\
\hline \multicolumn{5}{|c|}{ AO fracture classification } \\
\hline B3 & 2 & 1 & & No significant difference \\
\hline $\mathrm{C} 2$ & 1 & 4 & Pearson Chi-Square & \\
\hline C3 & 12 & 17 & 0.42 & \\
\hline \multicolumn{5}{|c|}{ Associated carpal injury } \\
\hline Osseous & 4 & 2 & Pearson Chi-Square & No significant difference \\
\hline Ligamentous & 3 & 8 & 0.25 & \\
\hline \multicolumn{5}{|l|}{ MWS } \\
\hline Mean & 89.00 & 89.09 & t-test & No significant difference \\
\hline Range & $65-100$ & $65-100$ & 0.98 & \\
\hline \multicolumn{5}{|l|}{$\overline{\text { Q DASH }}$} \\
\hline Mean & 15 & 22 & t-test & Significant difference \\
\hline Range & $0-36$ & $9-36$ & 0.018 & \\
\hline \multicolumn{5}{|l|}{ VAS } \\
\hline Mean & 0.59 & 3.53 & t-test & Significant difference \\
\hline Range & $0-3$ & $2-5$ & 0.0 & \\
\hline
\end{tabular}

MWS - Mayo Wrist Score, Q DASH - Quick Disabilities of Arm, Hand, and Shoulder Questionnaire, VAS - Visual Analogue Scale

\section{Discussion}

Operative treatment of distal radius fracture in these days is in majority of cases done through the volar approach and angular stable volar plates are used. This approach has several advantages. It is relatively straightforward and minimally disturbs local anatomical structures. Implant is placed in submuscular position and minimally interferes with adjacent flexor tendons. The result of this approach is a relatively low complications rate. Unfortunately, some of the distal radius fractures are difficult to be treated through volar approach and have to be done through the dorsal approach. Space available for the implant placement in dorsal approach is limited. Moreover, there is a close relation between the extensor tendons and dorsally placed implant. In current literature, published complications rates in dorsal radius plating are as high as $60 \%(19,27,28)$.

The results of our study indicated that dorsal plating in distal radius fractures had a high complication rate and the implant had to be removed frequently. In our study group, the implant removal was indicated in $51 \%$ of patients.

Sanchez and Jakubietz (19) mentioned that extensor tendon irritation was one of the most frequently reported complication. Tenosynovitis and extensor tendons ruptures had received an extensive attention in recent papers $(25,29,30,31)$. Tendinitis resulting in need for plate removal had been reported in up to $20 \%$ to 33 $\%$ of cases $(17,25)$. To prevent this complication, some authors suggested an early implant removal $(22,23)$. Others recommend a coverage of dorso-ulnar plate by retinacular flap (6). Chiang and Roach used this technique with limited success (20). In their review, 9 out of 20 patients ( $45 \%$ ) required a plate removal. At the time of implant removal, they reported that the extent of plate coverage by the retinacular flap created during initial surgery was not clearly apparent in all cases. They reported thinning or absence of the retinacular flap beneath the radial wrist extensors. Contrary to these findings, we did not observed retinacular flap problems in our study group.

Similar to Jakubietz (22), we assume that in most of the cases tendinitis resulted from hardware placement in narrow space between the dorsal cortex and extensor tendons. Despite the use of low-profile dorsal plates, which were designed to avoid tendon irritation problems (31), we have observed tendon related problems in several cases. Therefore, in patients with dorsal wrist pain or tendon irritation clinical findings, an ultrasound examination was performed to exclude tenosynovitis or partial ruptures of extensor tendons (33).

In patients whose plates were removed due to a superficial radial nerve neuropathy, we saw a good soft tissue coverage of radial plate, despite the fact that the first compartment was left open during the initial surgery. We assume that the superficial radial nerve irritation could be the result of soft tissue scarring around the radial plate. Another possible reason could be an unrecognized lesion at the time of trauma or secondary to the initial surgery.

The second most frequent complication in our study group that was the reason for implant removal, was a decreased range of motion of radio-carpal joint. In four of our patients during implant removal procedure, we noticed hyperplastic soft tissue reaction, probably as a reaction to the titanium implants. Cohen et al (34) identified no differences between titanium and stainless steel im- 
plants using in canine models. Keller et al (23) in their study group of 320 patients treated with dorsal plates observed less soft tissue reaction when using a steel plate compared to a titanium plate. In our patients after the hardware removal and the manipulation of radio-carpal joint under general or peripheral block anesthesia, the range of motion increased in all but one patient. Similarly, the pain level decreased. We believe that implant removal leads to a reduction of local irritation and inflammation, because of the avoidance of exposure of adjacent soft tissues to titanium alloy.

According to statistical analysis, there were no significant differences in demographic variables and Mayo wrist score rates between the groups with or without implant removal. Statistically significant differences were identified in subjective parameters Visual analogue scale and Quick DASH scores. As a result of statistical analysis, we consider these parameters as a significant predictors of the implant removal necessity. The dorsal wrist pain syndrome seems to be the main indicator of implant related soft tissue problems.

There are several potential limitations to this study. The first one is the length of the follow up. Despite the claims of Kreder et al (35) that one year of follow up is sufficient to allow conclusion about the final results of distal radius fracture treatment, and Shin and Jupiter (3) that most of tendon problem occurred before the end of 7 th month after the surgery, there is a report in literature on a delayed extensor tendon rupture after 7 years post trauma (36). Another possible limitation may be that surgical procedures were performed in a teaching hospital to which patients with complicated fractures were transferred from other hospitals in the region. So, the frequency of complications and implant removal should be carefully generalized as there is a referral bias in the cohort of patients.

\section{Conclusion}

Our study showed that there was no need for a routine implant removal after dorsal plating of distal radius fractures in patients without problems in follow up period. Dorsal wrist pain syndrome was the main indicator for implant related soft tissue problems. Restrictions in range of motion and extensor tendon related problems were the most frequent reason for plate removal. According to our statistical analysis, possible indicators for implant removal may be considered Visual analogue scale and Quick DASH scores. In our opinion, meticulous follow up is needed for patients with even a mild inconvenience after dorsal plating of distal radius fractures for early detection of soft tissue irritation and prevention of delayed tendon injury. In these cases, an early implant removal is indicated.

\section{References}

1. Court-Brown CM, Caesar B. Epidemiology of adult fractures: a review. Injury 2006; 37 (8): 691-697.

2. Harness NG, Meals RA. The history of fracture fixation of the hand and wrist. Clin Orthop Relat Res 2006; 445: 19-29.

3. Shin EK, Jupiter JB. Current concepts in the management of distal radius fractures. Acta Chir Orthop Traum Čech 2007; 74: 233-246.
4. Graham TJ. Surgical correction of malunited fractures of the distal radius. J Amer Acad Orthop Surg 1997; 5: 270-281.

5. Ring D, Jupiter JB, Brennwald J, Buchler U, Hastings H 2nd. Prospective multicenter trial of a plate for dorsal fixation of distal radius fractures. J Hand Surg Am 1997; 22 (5): 777-784.

6. Rikli DA et al. Dorsal double plate fixation of distal radial fractures. Operat Orthop Traumatol 2005; 17: 624-640.

7. Goldfarb CA, Rudzki JR, Catalano LW,Hughes M, Borrelli J Jr. Fifteen-year outcome of displaced intra-articular fractures of the distal radius. J Hand Surg Am 2006; 31: 633-639.

8. Kyle SB, Nance ML, Rutherford GW Jr, Winston FK. Skateboardassociated injuries: participation-based estimates and injury characteristics. J Trauma 2002; 53 (4): 686-690.

9. Garcia-Elias M, Folgar MA. The management of wrist injuries: an international perspective. Injury 2006; 37 (11): 1049-1056.

10. Karambouroglu GK, Axelrod TS. Complications of the AO/ASIF titanium distal radius plate system ( $\pi$ plate) in internal fixation of the distal radius: A brief report J Hand Surg Am 1998; 23: 737-741.

11. McQueen MM. Simpson D, Court-Brown CM. Use of the Hoffman 2 compact external fixator in the treatment of redisplaced unstable distal radial fracture. J Orthop Trauma, 1999; 13: 501-505.

12. Mittal R, Morley J, Dinopoulos H, Drakoulakis EG, Vermani E, Giannoudis PV. Use of bio-resorbable implants for stabilisation of distal radius fractures: The United Kingdom patients' perspective. Injury 2005; 36: $333-338$.

13. Rozental TD, Blazar PE. Functional outcome and complications after volar plating for dorsally displaced, unstable fractures of the distal radius. J Hand Surg Am 2006; 31: 359-365.

14. Ruch DS, Papadonikolakis A. Volar versus dorsal plating in the management of intra-articular distal radius fractures. J Hand Surg Am 2006; 31: $9-16$.

15. Handoll HHG, Madhok R. Surgical interventions for treating distal radial fractures in adults. Cochrane Database of Systematic Reviews 2009; 3. Art. No.: CD003209. doi:10.1002/14651858.CD 003209. pub2.

16. Jupiter JB, Marent-Huber M; LCP Study Group. Operative management of distal radial fractures with 2.4-millimeter locking plates. A multicenter prospective case series. J Bone Joint Surg Am 2009; 91 (1): 55-65.

17. Campbell DA. Open reduction and internal fixation of intraarticular and unstable fractures of the distal radius using the $\mathrm{AO}$ distal radius plate. J Hand Surg Br 2000; 25 (6): 528-534.

18. Vlcek M, Landor I, Horak Z, Musil V, Sosna A, Jonas D. Mathematical modelling for the comparison of plate and intramedullary osteosynthesis stability in intraarticular distal radius fractures. Bratisl Lek Listy 2014; 115: 107 - 111

19. Wei J, Yang TB, Luo W, Qin J B, Kong FJ. Complications following dorsal versus volar plate fixation of distal radius fracture: a meta-analysis. J Int Med Res 2013; 41 (2): 265-275.

20. Chiang PP, Roach S, Baratz ME. Failure of a retinacular flap to prevent dorsal wrist pain after titanium pi plate fixation of distal radius fractures. J Hand Surg Am 2002; 27: 724-728.

21. van Manen CJ, Dekker LM, van Eerten $P$ et al. Bio-resorbable versus metal implants in wrist fractures: a randomised trial. Arch Orthop Trauma Surg 2008; 128: 1413-1417. 


\section{$357-362$}

22. Jakubietz MG, Gruenert JG Jakubietz RG. Palmar and dorsal fixedangle plates in AO C-type. J Orthop Surg Res 2012; 7: 8-13.

23. Keller M, Steiger R. The (pi) plate: An implant for unstable extension fractures of the distal radius in patients with osteoporotic bone. Techniques Hand Upper Extremity Surg 2004; 8 (4): 212-218.

24. Sanchez T, Jakubietz M, Jakubietz R, Mayer J, Beutel FK, Grünert J. Complications after Pi Plate osteosynthesis. Plast Reconstr Surg 2005; 116 (1): 153-158.

25. Herron M, Faraj A, Craigen MA. Dorsal plating for displaced intraarticular fractures of the distal radius. Injury 2003; 34: 497-502.

26. Busam ML, Esther RJ, Obremskey WT. Hardware removal: indications and expectations. J Am Acad Orthop Surg 2006; 14 (2): 113-120.

27. Grewal R, Perey B, Wilmink $M$ et al. A randomised prospective study on the treatment of intraarticular distal radius fractures: open reduction and internal fixation with dorsal plating versus mini open reduction, percutaneous fixation, and external fixation. J Hand Surg Am 2005; 30: 764-772.

28. Jakubietz RG, Grünert JG, Kloss DF et al. A randomised clinical study comparing palmar and dorsal fixed-angle plates for the internal fixation of AO C-type fractures of the distal radius in the elderly. J Hand Surg Eur 2008; 33: 600-604.

29. McKay SD, MacDermid JC, Roth JH, Richards RS. Assessment of complications of distal radius fractures and development of a complication checklist. J Hand Surg 2001; 26A: 916 -922.
30. Lutsky K, Boyer, Goldfarb CH. Dorsal locked plate fixation of distal radius fractures. J Hand Surg 2013; 38A: 1414-1422.

31. Lowry KJ, Gainor BJ, Hoskins JS. Extensor tendon rupture secondary to the AO/ASIF titanium distal radius plate without associated plate failure: a case report. Am J Orthop 2000; 29: 789 -791.

32. Yu YR, Makhni MC, Tabrizi S, Rozental TD, Mundanthanam G, Day CS. Complications of low-profile dorsal versus volar locking plates in the distal radius: a comparative study. J Hand Surg Am 2011; 36 (7): 1135-1141.

33. Krettek C, Müller C, Meller R, Jagodzinski M,Hildebrand F, Gaulke R. Ist eine routinemäßige Implantatentfernung nach unfallchirurgischen Eingriffen sinnvoll? Unfallchirurg 2012; 115: 315-322.

34. Cohen MS, Turner TM, Urban RM. Effects of implant material and plate design on tendon function and morphology. Clin Orthop Relat Res 2006; 445: 81-90.

35. Kreder HJ, Hanel DP, Agel J et al. Indirect reduction and percutaneous fixation versus open reduction and internal fixation for displaced intraarticular fractures of the distal radius.J Bone Joint Surg Br 2005; 87 : 829-836.

36. Hattori Y, Doi K,Sakamoto S, Yukata K. Delayed rupture of extensor digitorum communis tendon following volar plating of distal radius fracture. Hand Surg 2008; 13: 183-185. 